\title{
The Positivity of Energy for Asymptotically Anti-de Sitter Spacetimes
}

\author{
E. Woolgar \\ Dept. of Mathematics, Statistics, and Computer Science \\ Dalhousie University \\ Halifax, Nova Scotia \\ Canada B3H 3J5
}

\begin{abstract}
We use the formulation of asymptotically anti-de Sitter boundary conditions given by Ashtekar and Magnon to obtain a coordinate expression for the general asymptotically AdeS metric in a neighbourhood of infinity. From this, we are able to compute the time delay of null curves propagating near infinity. If the gravitational mass is negative, so will be the time delay (relative to null geodesics at infinity) for certain null geodesics in the spacetime. Following closely an argument given by Penrose, Sorkin, and Woolgar, who treated the asymptotically flat case, we are then able to argue that a negative time delay is inconsistent with non-negative matter-energies in spacetimes having good causal properties. We thereby obtain a new positive mass theorem for these spacetimes. The theorem may be applied even when the matter flux near the boundary-at-infinity falls off so slowly that the mass changes, provided the theorem is applied in a time-averaged sense. The theorem also applies in certain spacetimes having local matter-energy that is sometimes negative, as can be the case in semi-classical gravity.
\end{abstract}




\section{The Positivity of Energy for Asymptotically Anti-de Sitter Spacetimes}

\section{Introduction}

Although most studies of the asymptotic properties of spacetime have considered the asymptotically flat case, there are several reasons to study spacetimes that asymptote to constant non-zero curvature manifolds, and in particular to the negative curvature case. The first reason is that the analysis is generally simpler. As we shall see, the boundaryat-infinity comes in one piece, unlike the asymptotically flat case with its two null pieces joined at the singular point $i^{0}$ representing spatial infinity. Furthermore, the negative cosmological constant acts as a mass term in the graviton equations of motion, so it is natural to impose boundary conditions that demand there be no gravitational radiation flux at infinity; in turn, this greatly simplifies the asymptotic metric.

An additional reason for studying asymptotic behaviour of this type is provided by supergravity. Certain physically interesting supergravity models require a negative cosmological constant, while other related models have scalar fields whose contribution to the energy can be negative. These models do not admit flat space as a solution (except when the scalar field potential vanishes); rather, anti-de Sitter space is the natural classical ground state (lowest energy classical solution) of these models. It is reasonable that anti-de Sitter space be a ground state - in particular, it has vanishing Weyl curvature, and mass-energy would be expected to vanish in conformally flat spacetimes. However, in the case of the supergravities with the scalar fields present, it was not known if the hamiltonians were bounded below. This served as motivation for the study of the stability of these models during the early 1980s. It was eventually shown that the dynamics of these theories implied that when the scalar field potentials were negative, the other fields were driven to positive energy configurations, such that the net energy was bounded below, provided the fields obeyed suitable boundary conditions. This result first emerged in a small perturbations analysis of Breitenlohner and Freedman. ${ }^{(1)}$ Abbott and Deser ${ }^{(2)}$ then gave a positive energy argument, based on supergravity but applied to pure general relativity, implying that the energy of anti-de Sitter space was a true lower bound in general relativity for all spaces obeying the appropriate boundary conditions. A full Witten-type ${ }^{(3)}$ proof for general relativity was provided by Gibbons, Hull, and Warner ${ }^{(4)}$, who also addressed the question of positivity of energy in the supergravity models. The issue of exactly what constituted "suitable boundary conditions" was resolved by Hawking. ${ }^{(5)}$ Ashtekar and Magnon ${ }^{(6)}$ reformulated these conditions and investigated the asymptotic properties of spacetimes obeying them.

Herein we adopt the Ashtekar and Magnon formulation, and derive from it a com-

pletely general coordinate form for the spacetime metric on a global neighbourhood of 
infinity. We then use this form of the metric to obtain a positive mass theorem that has no direct relation to supergravity (at least, none that is apparent), and in particular does not use spinorial methods in its derivation. Rather, we adapt to the present case the method of Penrose, Sorkin, and Woolgar, ${ }^{(7)}$ who recently obtained a positivity theorem for the mass of asymptotically flat spacetimes. This method depends on an analysis of the time delay effect for null geodesics moving under the influence of a gravitating mass. We show that the time delay due to a negative mass is incompatible with the geometric focussing effect (i.e. the gravitational lens effect, if one prefers) of generic curvature created by positive local matter-energy distributions.

In part, our motivation is to present the method of ref. (7), suitably modified, in a context which has fewer complications than are present in the asymptotically flat case. Secondly, it now appears that the method of ref. (7), suitably generalised, ${ }^{(8)}$ may actually yield somewhat different information in certain contexts than can be extracted from the spinorial proofs, leading to new Bogomol'nyi-type bounds on the mass and charges of fields. There is a third motivation. This treatment of anti-de Sitter space is a natural step in a programme that should next be directed to the study of asymptotically Robertson-Walker metrics, especially those with positive spatial curvature. It is remarkable that very little analytical work exists in the general Robertson-Walker case, considering especially that perturbations of FRW and de Sitter spacetimes are so important in the study of physical cosmology. For further motivation along similar lines, see ref. (9).

We follow the conventions of ref. (10), and use early latin letters as abstract indices, while greek indices refer to coordinate systems, and middle latin indices refer to certain directions within those coordinate systems. For a weaker version of the argument herein, which applies only to asymptotically AdeS-Schwarzschild spacetimes, see ref. (11).

\section{Asymptotic Coordinate System}

Here we restate what is sometimes known as Hawking's reflexive boundary condition, ${ }^{(5)}$ in the formulation of Ashtekar and Magnon. ${ }^{(6)}$

Definition 1: A spacetime $\left(\mathcal{M}, g_{a b}\right)$ is asymptotically anti-de Sitter if there exists a manifold-with-boundary $\tilde{\mathcal{M}}$, a $C^{3}$ metric $\tilde{g}_{a b}$ on $\tilde{\mathcal{M}}$, and a diffeomorphism from $\mathcal{M}$ onto $\tilde{\mathcal{M}} \backslash \partial \tilde{\mathcal{M}}$ such that

(i) there exists a $C^{3}$ function $\Omega$ on $\tilde{\mathcal{M}}$ such that $\tilde{g}_{a b}=\Omega^{2} g_{a b}$ on $\mathcal{M}$,

(ii) $\mathcal{I}=\partial \tilde{\mathcal{M}}$ has topology $S^{2} \times R$ and is defined as the surface $\Omega=0$,

(iii) $g_{a b}$ satisfies $R_{a b}-\frac{1}{2} g_{a b} R+\Lambda g_{a b}=8 \pi T_{a b}$, with $\Lambda<0$ (we choose units in which $\Lambda=-3$ ), where $\Omega^{-3} T_{a}^{b}$ admits a smooth limit to $\mathcal{I}$, and

(iv) the group of conformal isometries of $\left(\mathcal{I}, q_{a b}\right)$ is the anti-de Sitter group (or covering group thereof), $q_{a b}$ being the restriction of $\tilde{g}_{a b}$ to $\mathcal{I}$. 
Condition (ii) shows that we are considering spaces that are asymptotic to universal antide Sitter space, which is $R^{4}$ - often the time axis is quotiented by the integers to produce a spacetime with closed timelike curves. Although we will work with Definition 1, the positivity theorem we obtain will hold under the quotient. Fig. (1) shows (universal) AdeS spacetime embedded in the Einstein cylinder, which is a prototype for the above embedding. For another prototypical case, see the description of the AdeS-Schwarzschild solution given in Appendix 2.

In contrast with the asymptotically flat case, ${ }^{(12)}$ the smoothness conditions in this definition are probably appropriate. Because the cosmological constant affects small perturbations of the metric in the manner that a mass term would, it is reasonable to believe that outgoing radiation does not reach $\mathcal{I}$, so smooth generic initial data are unlikely to destroy smoothness at $\mathcal{I}$ at later times (although the author is not aware of specific results in the AdeS case). It is therefore likely that $C^{3}$ smoothness at $\mathcal{I}$ is a consistent assumption. Furthermore, it seems a necessary assumption because $\mathcal{I}$ is timelike (as we shall verify), and so inbound radiation flux at $\mathcal{I}$ is possible. Thus, asymptotically AdeS spaces are not globally hyperbolic, and would be unsuitable for many physical calculations unless the boundary conditions forbid such flux. Ref. (6) shows that the absence of Bondi flux at $\mathcal{I}$ is derivable from the above smoothness conditions.

Condition (iv) permits us to write the line element of $q_{a b}$ as

$$
-d t^{2}+d \theta^{2}+\sin ^{2} \theta d \phi^{2}
$$

We extend these coordinates to a global neighbourhood of $\mathcal{I}$ in any convenient way. The "physical" line element corresponding to $g_{a b}$ will be conformal to the warped product

$$
-d t^{2}+d \theta^{2}+\sin ^{2} \theta d \phi^{2}+\alpha d \xi^{2}+\mathcal{O}(f(\xi)),
$$

where $f(\xi)$ vanishes on $\mathcal{I}$. We use "ᄉ्=" to denote equality on $\mathcal{I}$, so the statement that $f$ vanishes on $\mathcal{I}$ is written as $f(\xi) \hat{=} 0$. Here there are no cross-terms $(d \xi d t, d \xi d \theta$, or $d \xi d \phi)$ on $\mathcal{I}$ because we define $\xi$ such that $\mathcal{I}$ is a level surface on which $t, \theta$, and $\phi$ serve as coordinates - if $\frac{\partial}{\partial \xi}$ is "metric-dual" to $d \xi$, it must then be orthogonal to $\mathcal{I}$.

Without loss of generality, we can thus require that the physical line element be conformal to

$$
-d t^{2}+\left(1-\Omega^{2}\right)\left(d \theta^{2}+\sin ^{2} \theta d \phi^{2}\right)+\beta \frac{d \Omega^{2}}{\left(1-\Omega^{2}\right)}+\mathcal{O}(\Omega),
$$

where $\Omega=\Omega(\xi) \hat{=} 0$ and where $\beta(\Omega)=\alpha(\xi)$. In particular, we take $\Omega=\cos \xi$, with $\mathcal{I}$ defined by $\xi=\frac{\pi}{2}$. Invoking (i) and (ii) above gives the physical line element

$$
d s^{2}=\Omega^{-2}\left(-d t^{2}+\beta \frac{d \Omega^{2}}{\left(1-\Omega^{2}\right)}+\left(1-\Omega^{2}\right)\left(d \theta^{2}+\sin ^{2} \theta d \phi^{2}\right)+\mathcal{O}(\Omega)\right) .
$$


Next, let us write the Einstein equation in terms of the quantity

$$
S_{a b}=R_{a b}-\frac{1}{6} g_{a b} R
$$

since $S_{a b}$ has somewhat simpler properties under conformal transformations than the Einstein tensor. The Einstein equation with cosmological constant (normalised so that $\Lambda=-3$; this can always be achieved by rescaling the coordinates) can be written as

$$
S_{a b}+g_{a b}=8 \pi g_{a c}\left(T_{b}^{c}-\frac{1}{3} \delta_{b}^{c} T\right)=8 \pi g_{a c} \mathcal{T}_{b}^{c}
$$

Let us now define $\tilde{S}_{a b}$ to be given from equation (5), but with $g_{a b}$ and its Ricci tensor replaced by $\tilde{g}_{a b}$ and its Ricci tensor. The conformal transformation property alluded to above is

$$
S_{a b}=\tilde{S}_{a b}+\frac{2}{\Omega} \tilde{\nabla}_{a} \tilde{\nabla}_{b} \Omega-\frac{1}{\Omega^{2}} \tilde{g}_{a b} \tilde{g}^{c d} \tilde{\nabla}_{c} \Omega \tilde{\nabla}_{d} \Omega
$$

where $\tilde{\nabla}_{a} \tilde{g}_{b c}=0$. Hence the field equation becomes

$$
\tilde{S}_{a b}+\frac{1}{\Omega^{2}} \tilde{g}_{a b}\left(1-\tilde{g}^{c d} \tilde{\nabla}_{c} \Omega \tilde{\nabla}_{d} \Omega\right)+\frac{2}{\Omega} \tilde{\nabla}_{a} \tilde{\nabla}_{b} \Omega=\frac{8 \pi}{\Omega^{2}} \tilde{g}_{a c} \mathcal{T}_{b}^{c}
$$

Given the smoothness requirements on $\tilde{g}_{a b}$, then $\tilde{S}_{a b}$ is finite at $\mathcal{I}$ and, given the fall-off condition (iii) on the stress tensor, the right-hand-side of equation $(8)$ is $\mathcal{O}(\Omega)$, so we obtain from the field equation $(8)$ the usual result ${ }^{(13)}$ that $\mathcal{I}$ has spacelike normal $n_{a}=\tilde{\nabla}_{a} \Omega$.

$$
\tilde{g}^{a b} \tilde{\nabla}_{a} \Omega \tilde{\nabla}_{b} \Omega=\tilde{g}^{a b} n_{a} n_{b} \hat{=} 1
$$

From this, we get that $\tilde{g}_{11} \hat{=} \beta \hat{=} 1$, since $\tilde{g}_{1 i} \hat{=} 0$, where from here onward $i \in\{0,2,3\}$ denotes the coordinates $(t, \theta, \phi)$. All this is of course standard, ${ }^{(14)}$ including the next step, which is to use the gauge freedom available in the choice of $\Omega$ to set

$$
\Omega^{-1}\left(\tilde{g}^{a b} n_{a} n_{b}-1\right) \hat{=} 0
$$

whence the field equation, the differentiability of $\tilde{g}_{a b}$, and the fall-off condition (iii) imply that

$$
\tilde{\nabla}_{a} \tilde{\nabla}_{b} \Omega \hat{=} 0
$$

This can also be written in our coordinates as $\tilde{\Gamma}_{a b}^{1} \hat{=} 0$, and it implies that $\tilde{g}_{11,1} \hat{=} 0$ and $\tilde{g}_{i j, 1} \hat{=} 0$, so we have eliminated all $\mathcal{O}(\Omega)$ terms except those in $\tilde{g}_{1 i}$. However, the coordinate transformation

$$
\begin{aligned}
x^{i} \longrightarrow \bar{x}^{i} & =x^{i}+\Omega^{2} f^{i}\left(x^{j}\right) \\
d x^{i} \longrightarrow d \bar{x}^{i} & =d x^{i}+2 \Omega f^{i}\left(x^{j}\right) d \Omega+\mathcal{O}\left(\Omega^{2}\right), \\
\Rightarrow d \bar{x}^{i} d \bar{x}^{j} & =d x^{i} d x^{j}+4 \Omega f^{(i} d x^{j)}+\mathcal{O}\left(\Omega^{2}\right)
\end{aligned}
$$


will remove any such terms at the expense only of new terms of $\mathcal{O}\left(\Omega^{2}\right)$, and so we may choose the coordinates so no $\mathcal{O}(\Omega)$ terms appear in the conformal metric, which takes the general form

$$
\begin{aligned}
d \tilde{s}^{2}= & -d t^{2}+\frac{d \Omega^{2}}{\left(1-\Omega^{2}\right)}+\left(1-\Omega^{2}\right)\left(d \theta^{2}+\sin ^{2} \theta d \phi^{2}\right) \\
& +\Omega^{2}\left(A d t^{2}+B d \Omega^{2}+C d \theta^{2}+D \sin ^{2} \theta d \phi^{2}\right) \\
& +2 \Omega^{2}(L d t d \theta+O d t d \phi+G d \theta d \phi) \\
& +2 \Omega^{2}(X d t+Y d \theta+Z d \phi) d \Omega \\
& +\Omega^{3}\left(J d t^{2}+K d \Omega^{2}+N d \theta^{2}+F \sin ^{2} \theta d \phi^{2}\right) \\
& +2 \Omega^{3}(M d t d \theta+S d t d \phi+Q d \theta d \phi) \\
& +2 \Omega^{3}(U d t+V d \theta+W d \phi) d \Omega+\mathcal{O}\left(\Omega^{4}\right) .
\end{aligned}
$$

The coefficients $B$ and $K$ in the above metric can be cancelled by the coordinate transformation

$$
\Omega=\bar{r}-\frac{1}{6} B \bar{r}^{3}-\frac{1}{8} K \bar{r}^{4}
$$

Hence

$$
d \Omega=\left(1-\frac{B}{2} \bar{r}^{2}-\frac{1}{2} K \bar{r}^{3}\right) d \bar{r}-\frac{1}{6} \bar{r}^{3} \sum_{i \neq 1} \frac{\partial B}{\partial x^{i}} d x^{i}+\mathcal{O}\left(\bar{r}^{4}\right)
$$

In addition to eliminating $B$ and $K$, this changes three other metric coefficients, the changes being effected by

$$
\begin{aligned}
& U \longrightarrow \bar{U}=U-\frac{1}{6} \frac{\partial B}{\partial t}, \\
& V \longrightarrow \bar{V}=V-\frac{1}{6} \frac{\partial B}{\partial \theta}, \\
& W \longrightarrow \bar{W}=W-\frac{1}{6} \frac{\partial B}{\partial \phi}
\end{aligned}
$$

Furthermore, we may make the coordinate transformation ${ }^{(15)}$

$$
x^{i}=\bar{x}^{i}-\frac{1}{3} \bar{r}^{3} F^{i}\left(\bar{x}^{j}\right)-\frac{1}{4} \bar{r}^{4} G^{i}\left(\bar{x}^{j}\right)
$$


where $x^{i} \in\{t, \theta, \phi\}$ and $\bar{x}^{i}=\{\bar{t}, \bar{\theta}, \bar{\phi}\}$. This produces the conformal metric

$$
\begin{aligned}
d \tilde{s}^{2}= & -d \bar{t}^{2}+\frac{d \bar{r}^{2}}{\left(1-\bar{r}^{2}\right)}+\left(1-\bar{r}^{2}\right)\left(d \bar{\theta}^{2}+\sin ^{2} \bar{\theta} d \bar{\phi}^{2}\right) \\
& +\bar{r}^{2}\left(A d \bar{t}^{2}+C d \bar{\theta}^{2}+D \sin ^{2} \bar{\theta} d \bar{\phi}^{2}\right) \\
& +2 \bar{r}^{2}(L d \bar{t} d \bar{\theta}+O d \bar{t} d \bar{\phi}+G d \bar{\theta} d \bar{\phi}) \\
& +2 \bar{r}^{2}\left[\left(X+F^{0}\right) d \bar{t}+\left(Y-F^{2}\right) d \bar{\theta}+\left(Z-F^{3} \sin ^{2} \bar{\theta}\right) d \bar{\phi}\right] d \bar{r} \\
& +\bar{r}^{3}\left[\left(J+\frac{2}{3} \frac{\partial F^{0}}{\partial \bar{t}}\right) d \bar{t}^{2}+\left(N-\frac{2}{3} \frac{\partial F^{2}}{\partial \bar{\theta}}\right) d \bar{\theta}^{2}\right. \\
& \left.\quad+\left(F-\frac{2}{3} \frac{\partial F^{3}}{\partial \bar{\phi}}-\frac{2}{3} F^{2} \cot \bar{\theta}\right) \sin ^{2} \bar{\theta} d \bar{\phi}^{2}\right] \\
& +2 \bar{r}^{3}\left[\left(M+\frac{1}{3} \frac{\partial F^{0}}{\partial \bar{\theta}}-\frac{1}{3} \frac{\partial F^{2}}{\partial \bar{t}}\right) d \bar{t} d \bar{\theta}+\left(S+\frac{1}{3} \frac{\partial F^{0}}{\partial \bar{\phi}}-\frac{1}{3} \frac{\partial F^{3}}{\partial \bar{t}} \sin ^{2} \bar{\theta}\right) d \bar{t} d \bar{\phi}\right. \\
& \left.\quad+\left(Q-\frac{1}{3} \frac{\partial F^{2}}{\partial \bar{\phi}}-\frac{1}{3} \frac{\partial F^{3}}{\partial \bar{\theta}} \sin ^{2} \bar{\theta}\right) d \bar{\theta} d \bar{\phi}\right] \\
& +2 \bar{r}^{3}\left[\left(\bar{U}+G^{0}\right) d \bar{t}+\left(\bar{V}-G^{2}\right) d \bar{\theta}+\left(\bar{W}-G^{3} \sin ^{2} \bar{\theta}\right) d \bar{\phi}\right] d \bar{r}+\mathcal{O}\left(\bar{r}^{4}\right),
\end{aligned}
$$

where the arguments of all functions appearing in this metric are the barred coordinates $\bar{x}^{i} \in\{\bar{t}, \bar{\theta}, \bar{\phi}\}$. We choose the functions $F^{i}$ and $G^{i}$ to satisfy

$$
\begin{aligned}
0 & =X+F^{0}=Y-F^{2}=Z-F^{3} \sin ^{2} \bar{\theta} \\
& =\bar{U}+G^{0}=\bar{V}-G^{2}=\bar{W}-G^{3} \sin ^{2} \bar{\theta},
\end{aligned}
$$

and define

$$
\begin{aligned}
\bar{M} & =M+\frac{1}{3} \frac{\partial F^{0}}{\partial \bar{\theta}}-\frac{1}{3} \frac{\partial F^{2}}{\partial \bar{t}} \\
& =M-\frac{1}{3} \frac{\partial X}{\partial \bar{\theta}}-\frac{1}{3} \frac{\partial Y}{\partial \bar{t}},
\end{aligned}
$$

and likewise make the obvious definitions for $\bar{S}, \bar{Q}, \bar{J}, \bar{N}$, and $\bar{F}$. After this, the conformal metric simplifies to

$$
\begin{aligned}
d \tilde{s}^{2}= & -d \bar{t}^{2}+\frac{d \bar{r}^{2}}{\left(1-\bar{r}^{2}\right)}+\left(1-\bar{r}^{2}\right)\left(d \bar{\theta}^{2}+\sin ^{2} \bar{\theta} d \bar{\phi}^{2}\right) \\
& +\bar{r}^{2}\left(A d \bar{t}^{2}+C d \bar{\theta}^{2}+D \sin ^{2} \bar{\theta} d \bar{\phi}^{2}\right) \\
& +2 \bar{r}^{2}(L d \bar{t} d \bar{\theta}+O d \bar{t} d \bar{\phi}+G d \bar{\theta} d \bar{\phi}) \\
& +\bar{r}^{3}\left(\bar{J} d \bar{t}^{2}+\bar{N} d \bar{\theta}^{2}+\bar{F} \sin ^{2} \bar{\theta} d \bar{\phi}^{2}\right) \\
& +2 \bar{r}^{3}(\bar{M} d \bar{t} d \bar{\theta}+\bar{S} d \bar{t} d \bar{\phi}+\bar{Q} d \bar{\theta} d \bar{\phi})+\mathcal{O}\left(\bar{r}^{4}\right)
\end{aligned}
$$

while the physical metric is

$$
d s^{2}=\Omega^{-2} d \tilde{s}^{2} .
$$


The next step is to compute the left-hand-side of the field equation (8) using the conformal metric (20). This task was performed both by hand and by the RCLASSI computer program for relativity calculations. The resulting field equations are

$$
\begin{aligned}
\frac{4}{3} A-B-\frac{1}{3} C-\frac{1}{3} D+\mathcal{O}(\bar{r}) & =8 \pi \mathcal{T}_{00}, \\
L+\mathcal{O}(\bar{r}) & =8 \pi \mathcal{T}_{02}, \\
O+\mathcal{O}(\bar{r}) & =8 \pi \mathcal{T}_{03}, \\
\frac{2}{3}(A-C-D)-B+\mathcal{O}(\bar{r}) & =8 \pi \mathcal{T}_{11}, \\
-\frac{1}{3} A+B+\frac{4}{3} C+\frac{1}{3} D+\mathcal{O}(\bar{r}) & =8 \pi \mathcal{T}_{22}, \\
G+\mathcal{O}(\bar{r}) & =8 \pi \mathcal{T}_{23}, \\
\left(-\frac{1}{3} A+B+\frac{1}{3} C+\frac{4}{3} D\right) \sin ^{2} \bar{\theta}+\mathcal{O}(\bar{r}) & =8 \pi \mathcal{T}_{33},
\end{aligned}
$$

where we define

$$
\mathcal{T}_{a b}=g_{a c} \mathcal{T}_{b}^{c}=\Omega^{-2} \tilde{g}_{a c} \mathcal{T}_{b}^{c}=\mathcal{O}(\Omega)=\mathcal{O}(\bar{r})
$$

according to the specified fall-off condition (iii). Three components of the field equations are not written above because their left-hand-sides had no $\mathcal{O}(1)$ contributions. We see that we must have

$$
L=O=G=0 \quad, \quad A=\frac{1}{2} B \quad, \quad C=D=-\frac{1}{2} B,
$$

and hence the conformal metric may be written as

$$
\begin{aligned}
d \tilde{s}^{2}= & -d \bar{t}^{2}+\frac{d \bar{r}^{2}}{\left(1-\bar{r}^{2}\right)}+\left(1-\bar{r}^{2}\right)\left(d \bar{\theta}^{2}+\sin ^{2} \bar{\theta} d \bar{\phi}^{2}\right) \\
& +\frac{B}{2} \bar{r}^{2}\left(d \bar{t}^{2}-d \bar{\theta}^{2}-\sin ^{2} \bar{\theta} d \bar{\phi}^{2}\right)+\left(J d \bar{t}^{2}+N d \bar{\theta}^{2}+F \sin ^{2} \bar{\theta} d \bar{\phi}^{2}\right. \\
& +2 M d \bar{t} d \bar{\theta}+2 S d \bar{t} d \bar{\phi}+2 Q d \bar{\theta} d \bar{\phi}) \bar{r}^{3}+\mathcal{O}\left(\bar{r}^{4}\right) .
\end{aligned}
$$

If we let

$$
d \tilde{s}_{\mathrm{AdeS}}^{2}=-d \bar{t}^{2}+d \bar{\xi}^{2}+\sin ^{2} \bar{\xi}\left(d \bar{\theta}^{2}+\sin ^{2} \bar{\theta} d \bar{\phi}^{2}\right),
$$

then the conformal metric can be written as

$$
\begin{aligned}
d \tilde{s}^{2}= & \left(1-\frac{1}{2} B \cos ^{2} \bar{\xi}\right) d \tilde{s}_{\text {AdeS }}^{2}+\frac{1}{2} B \cos ^{2} \bar{\xi} d \bar{\xi}^{2} \\
& +\cos ^{3} \bar{\xi}\left(\bar{J} d \bar{t}^{2}+\bar{N} d \bar{\theta}^{2}+\bar{F} \sin ^{2} \bar{\theta} d \bar{\phi}^{2}\right) \\
& +2 \cos ^{3} \bar{\xi}(\bar{M} d \bar{t} d \bar{\theta}+\bar{S} d \bar{t} d \bar{\phi}+\bar{Q} d \bar{\theta} d \bar{\phi}) \\
& +\mathcal{O}\left(\cos ^{4} \bar{\xi}\right) .
\end{aligned}
$$


Purely as an aside, we take the opportunity to recalculate the field equations using the metric (27) in order to examine the $\mathcal{O}(\bar{r})$ terms. They reduce to the statements that $\mathcal{T}_{\mu \nu}=\mathcal{O}\left(\bar{r}^{2}\right)$ for $\mu \neq \nu$, and that each of the diagonal components of $\mathcal{T}_{a b}$ are proportional to the combination $(\bar{J}-\bar{N}-\bar{F}-\bar{K}) \bar{r}$, up to correction terms which are $\mathcal{O}\left(\bar{r}^{2}\right)$. Thus, all the off-diagonal components of $\mathcal{T}_{a b}$ vanish at this order, and all the diagonal components will as well provided the trace vanishes, no doubt a consequence of the asymptotic symmetry. Ashtekar and Magnon alluded to the fact that the Bianchi identities and the $\mathcal{O}(\bar{r})$ fall-off condition on $\mathcal{T}_{a b}$ is strong enough to imply that many of the components of $\mathcal{T}_{a b}$ actually fall off as $\mathcal{O}\left(\bar{r}^{2}\right)$. Note, however, that we do not require any condition on $\bar{J}-\bar{N}-\bar{F}-\bar{K}$ in what follows, and so the diagonal components of $\mathcal{T}_{a b}$ need not vanish at $\mathcal{O}(\bar{r})$ here; we do not make any use of the $\mathcal{O}(\bar{r})$ field equations.

The Weyl tensor of this metric, to $\mathcal{O}(\bar{r})$ inclusive, is

$$
\begin{array}{cc}
C_{0101}=-\left(\bar{J}+\frac{1}{2} \bar{N}+\frac{1}{2} \bar{F}\right) \bar{r}, & C_{0112}=\frac{3}{2} \bar{M} \bar{r} \\
C_{0113}=\frac{3}{2} \bar{S} \bar{r}, & C_{0202}=\left(\bar{F}+\frac{1}{2} \bar{J}-\frac{1}{2} \bar{N}\right) \bar{r}, \\
C_{0203}=-\frac{3}{2} \bar{Q} \bar{r}, & C_{0223}=-\frac{3}{2} \bar{S} \bar{r}, \\
C_{0303}=\left(\bar{N}+\frac{1}{2} \bar{J}-\frac{1}{2} \bar{F}\right) \bar{r} \sin ^{2} \bar{\theta} & C_{0323}=\frac{3}{2} \bar{M} \bar{r} \sin ^{2} \bar{\theta}, \\
C_{1212}=\left(\frac{1}{2} \bar{F}-\frac{1}{2} \bar{J}-\bar{N}\right) \bar{r} & C_{1213}=-\frac{3}{2} \bar{Q} \bar{r}, \\
C_{1313}=\left(\frac{1}{2} \bar{N}-\frac{1}{2} \bar{J}-\bar{F}\right) \bar{r} \sin ^{2} \bar{\theta} & C_{2323}=\left(\frac{1}{2} \bar{F}+\frac{1}{2} \bar{N}+\bar{J}\right) \bar{r} \sin ^{2} \bar{\theta}
\end{array}
$$

with the other independent components being zero at this order. Since there is no component $C_{1 i j k}$ at this order (where $i, j, k \in\{0,2,3\}$ ), the (rescaled) magnetic part of the Weyl tensor vanishes on $\mathcal{I}$. According to Ashtekar and Magnon, this is precisely the statement that the metric satisfies Hawking's "reflexive" boundary condition. Furthermore, note that the (rescaled) electric part of the Weyl tensor, defined by

$$
E_{c d}=\Omega^{-1} C_{a c b d} n^{a} n^{b} \quad, \quad n^{a}=\tilde{g}^{a b} \tilde{\nabla}_{b} \Omega,
$$

has its non-vanishing components on $\mathcal{I}$ given as

$$
\begin{array}{rr}
E_{00} \hat{=}-\left(\bar{J}+\frac{1}{2} \bar{N}+\frac{1}{2} \bar{F}\right), & E_{02} \hat{=}-\frac{3}{2} \bar{M}, \\
E_{22} \hat{=} \frac{1}{2} \bar{F}-\frac{1}{2} \bar{J}-\bar{N}, & E_{03} \hat{=}-\frac{3}{2} \bar{S}, \\
E_{33} \hat{=}\left(\frac{1}{2} \bar{N}-\frac{1}{2} \bar{J}-\bar{F}\right) \sin ^{2} \bar{\theta} \quad, & E_{23} \hat{=}-\frac{3}{2} \bar{Q} .
\end{array}
$$

There cannot, of course, be any non-zero $E_{1 \mu}$ component. 
Given the above construction, we may state the following result.

Proposition 1: If $\left(\mathcal{M}, g_{a b}\right)$ is asymptotically anti-de Sitter, there exist coordinates $(t, \xi, \theta, \phi)$ on $\mathcal{U} \cup \mathcal{I}$, where $\mathcal{I} \subseteq \overline{\mathcal{U}}$ (the closure of $\mathcal{U}$ ) and $\mathcal{I}$ is the surface $\xi=\frac{\pi}{2}$, in which the metric is conformal to

$$
\begin{aligned}
d \tilde{s}^{2}=(1- & \left.\frac{1}{2} B \cos ^{2} \bar{\xi}-\frac{1}{3}(\bar{J}-\bar{N}-\bar{F}) \cos ^{3} \bar{\xi}\right) d \tilde{s}_{\text {AdeS }}^{2} \\
& +\left(\frac{1}{2} B+\frac{1}{3}(\bar{J}-\bar{N}-\bar{F}) \cos \bar{\xi}\right) \cos ^{2} \bar{\xi} d \bar{\xi}^{2} \\
& -\frac{2}{3}\left(\cos ^{3} \bar{\xi}\right) E_{i j} d \bar{x}^{i} d \bar{x}^{j}+\mathcal{O}\left(\cos ^{4} \bar{\xi}\right),
\end{aligned}
$$

with $d \tilde{s}_{\text {AdeS }}^{2}$ given by (26), and where the $E_{i j}$ are the components in this coordinate system of the electric part of the Weyl tensor (rescaled by $\Omega^{-1}$ ) on $\mathcal{I}$, extended into spacetime by Lie dragging along lines of constant $\bar{t}, \bar{\theta}$, and $\bar{\phi}$.

The deviation of the metric from the anti-de Sitter metric is described at leading order essentially by the electric components of the Weyl tensor, which is natural because of the absence of gravitational radiation at infinity. The above metric suffers from the usual coordinate singularities of the polar coordinates $(\bar{\theta}, \bar{\phi})$ of course, so at least two charts are needed to cover $\mathcal{U}$ with good coordinates - this is an artifact of the $S^{2} \times R$ topology of $\mathcal{I}$. However, this sort of difficulty is so mild that it is convenient simply to ignore it in what follows.

\section{The Time Delay Formula}

Consider now the conformal metric. Along any null curve, not necessarily geodesic, we have

$$
\begin{aligned}
0 & =\frac{d \tilde{s}}{1-H \cos ^{2} \xi} \\
& =d \tilde{s}_{\text {AdeS }}^{2}+H \cos ^{2} \xi d \xi^{2}-\frac{2}{3}\left(\cos ^{3} \xi\right) E_{i j} d x^{i} d x^{j}+\mathcal{O}\left(\cos ^{4} \xi\right)
\end{aligned}
$$

where

$$
H=\frac{1}{2} B+\frac{1}{3}(J-N-F) \cos \xi .
$$

Throughout this section and the next, we drop the bars over the coordinates and over the functions $J, N$, etc., and so (32) implies that

$$
\begin{aligned}
0=-d t^{2} & +d \xi^{2}+\sin ^{2} \xi\left(d \theta^{2}+\sin ^{2} d \phi^{2}\right)+H \cos ^{2} \xi d \xi^{2} \\
& -\frac{2}{3}\left(\cos ^{3} \xi\right) E_{\mu \nu} d x^{\mu} d x^{\nu}+\mathcal{O}\left(\cos ^{4} \xi\right)
\end{aligned}
$$


whence of course

$$
d t^{2}=d \sigma^{2}+H \cos ^{2} \xi d \xi^{2}-\frac{2}{3}\left(\cos ^{3} \xi\right) E_{\mu \nu} d x^{\mu} d x^{\nu}+\mathcal{O}\left(\cos ^{4} \xi\right)
$$

where

$$
d \sigma^{2}=d \xi^{2}+\sin ^{2} \xi\left(d \theta^{2}+\sin ^{2} \theta d \phi^{2}\right)
$$

is the "round metric" on $S^{3}$. We are not interested in the whole 3 -sphere, but rather a region within the hemisphere $\xi \in\left[0, \frac{\pi}{2}\right]$, which represents a spatial slice of anti-de Sitter space, with $\xi=\frac{\pi}{2}$ being infinity.

Now consider the "neighbourhood of infinity" $\mathcal{U}$ on which we are working. It is not $S^{3} \times R$, because it contains neither points $\xi \geq \frac{\pi}{2}$ nor points $\xi \leq \frac{\pi}{2}-\epsilon$, for some $\epsilon>0$. Rather, the neighbourhood can be described as $\mathcal{U}=\mathcal{T} \times R$, where $\mathcal{T}$ is the 3-dimensional manifold $\left\{x \mid \xi \in\left(\frac{\pi}{2}-\epsilon, \frac{\pi}{2}\right), \forall \theta, \forall \phi\right\}$; essentially $\mathcal{T}$ is a 3 -dimensional version of the Tropic of Capricorn (taking $\xi$ to be co-latitutude defined using the South Pole - see figs. $(2,3)$ ). Then $\sigma$ defines an arc length on $\mathcal{T}$ with respect to the metric (36). The idea is to use this arc length to parametrise null curves in $\mathcal{Q}=\mathcal{U} \cup \mathcal{I}$. Along such curves, we have

$$
\begin{aligned}
d t & = \pm\left[1+H\left(\frac{d r}{d \sigma}\right)^{2} \cos ^{2} \xi-\frac{2}{3}\left(\cos ^{3} \xi\right) E_{\mu \nu} \frac{d x^{\mu}}{d \sigma} \frac{d x^{\nu}}{d \sigma}+\mathcal{O}\left(\cos ^{4} \xi\right)\right]^{1 / 2} d \sigma \\
& = \pm\left[1+\frac{1}{2} H\left(\frac{d r}{d \sigma}\right)^{2} \cos ^{2} \xi-\frac{1}{3}\left(\cos ^{3} \xi\right) E_{\mu \nu} \frac{d x^{\mu}}{d \sigma} \frac{d x^{\nu}}{d \sigma}+\mathcal{O}\left(\cos ^{4} \xi\right)\right] d \sigma
\end{aligned}
$$

In particular, an arbitrary $C^{0}$ and piecewise $C^{1}$ future-null curve that leaves $p \in \mathcal{I}$ and arrives at $q \in \mathcal{I}$, having traversed arc length $\sigma_{p q}$, will reach its destination at coordinate time

$$
t_{q}=t_{p}+\sum_{i=0}^{n} \int_{\sigma_{i}}^{\sigma_{i+1}}\left(1+\frac{1}{2}\left(\cos ^{2} \xi\right) H\left(\frac{d r}{d \sigma}\right)^{2}-\frac{1}{3}\left(\cos ^{3} \xi\right) E_{\mu \nu} \frac{d x^{\mu}}{d \sigma} \frac{d x^{\nu}}{d \sigma}+\mathcal{O}\left(\cos ^{4} \xi\right)\right) d \sigma
$$

assuming $n$ points at whicch the curve fails to be differentiable.

Let $\gamma$ be a null geodesic ruling $\mathcal{I}$ from $P$ to $Q .{ }^{*}$ We vary this curve according to a very particular set of conditions, similar to those of ref. (16):

* It may seem surprising at first, but indeed this timelike $\mathcal{I}$ admits null generators which are geodesics of $d \widetilde{s}^{2}$ - in fact, it admits many families of them, about which more will be said shortly. Indeed, since equation (40) will show the first-order (but clearly not thirdorder) stability of the arrival time of $\gamma$ under the variation discussed here, the methods of ref. (16) verify that $\gamma$ is geodesic, given that it is null and lies on $\mathcal{I}$. 
(i) The varied curves $\gamma^{\prime}$ start at $P$ and end on the same generator of $\mathcal{I}$ (i.e. the same integral curve of $\frac{\partial}{\partial t}$ on $\mathcal{I}$ ) as contains $Q$, but they reach this generator at points $q$, where $t_{q}$ need not equal $t_{Q}$.

(ii) The varied curves remain null in $d \tilde{s}^{2}$ and are $C^{0}$, piecewise $C^{1}$, so the time-of-flight formula (38) applies to all these curves.

(iii) Each varied curve projects to a geodesic on $S^{3}$ with the metric $d \sigma^{2}$ - hence they each traverse arc length $\pi$ as measured by $\sigma$.

(iv) Along any varied curve $\gamma^{\prime}$, if we denote $\gamma^{\prime \mu}(\sigma)=\frac{d x^{\mu}(\sigma)}{d \sigma}$ as giving the tangent components and if we denote $\gamma^{\mu}(\sigma)$ as the tangent components for $\gamma$ at the same parameter value $\sigma$, then

$$
\gamma^{\prime \mu}(\sigma)=\gamma^{\mu}(\sigma)+\mathcal{O}(\cos \xi)
$$

(v) All varied curves remain within the region $\mathcal{Q}$, whence along each curve we have $\xi(\sigma) \in$ $\left[\frac{\pi}{2}-\epsilon, \frac{\pi}{2}\right], \forall \sigma$.

In particular, condition (iv) implies that $d r / d \sigma=\mathcal{O}(\cos \xi)$, and so the time-of-flight along any curve obeying the above conditions is

$$
t_{q}-t_{P}=\pi-\frac{1}{3} \cos ^{3} \xi_{0} \int_{0}^{\pi}\left(E_{\mu \nu} \gamma^{\mu} \gamma^{\nu}+\mathcal{O}(\cos \xi)\right) d \sigma
$$

for some $\xi_{0} \in\left(\frac{\pi}{2}-\epsilon, \frac{\pi}{2}\right)$. The remaining integral is taken along the curve $\gamma \subseteq \mathcal{I}$, as this will induce only errors of $\mathcal{O}(\epsilon)$ in the integral, and need no longer be broken, as $\gamma$ is smooth. In other words, the integral in the following expression is to be taken along a curve lying on the metrical (i.e. round) 2 -sphere $\xi=\frac{\pi}{2}$.

$$
t_{q}-t_{P}=\pi-\frac{1}{3} \cos ^{3} \xi_{0} \int_{0}^{\pi}\left(E_{\mu \nu} \gamma^{\mu} \gamma^{\nu}+\mathcal{O}(\epsilon)\right) d \sigma
$$

Finally, the time-delay (possibly negative) of the null curve $\gamma^{\prime}$ compared to that of $\gamma$ is

$$
\Delta t=\left.\left(t_{q}-t_{P}\right)\right|_{\gamma^{\prime}}-\left.\left(t_{Q}-t_{P}\right)\right|_{\gamma}=-\frac{1}{3} \cos ^{3} \xi_{0}\left(\int_{0}^{\pi} E_{\mu \nu} \gamma^{\mu} \gamma^{\nu} d \sigma+\mathcal{O}(\epsilon)\right)
$$

For a pictorial description of the variation and the time delay effect, see figs. (2-4).

Consider the case wherein the time-delay is negative. This will mean that the "fastest curve" from $P$ to a given timelike generator of $\mathcal{I}$ will not be one that remains always on $\mathcal{I}$ - it will pass through spacetime - provided of course such a fastest curve exists. We will derive arguments why such a fastest curve cannot exist off $\mathcal{I}$, implying that the time 
delay is always non-negative. This implies a non-negativity condition on the integral in equation (42), which leads to a positive mass.

\section{The Mass and Geodesic Focussing}

In this section, we will prove the following theorem:

Theorem 2: Let $\left(\mathcal{M}, g_{a b}\right)$ be an asymptotically anti-de Sitter spacetime obeying the Borde energy condition and having a boundary-at-infinity $\mathcal{I}$ such that, $\forall p \in \mathcal{I}$, there is a 2 -sphere cross-section of $\mathcal{I}$ that is not in the causal future of $p$ ** $^{* *}$ Let $t$, parametrising the timelike Killing field $\frac{\partial}{\partial t}$ on $\mathcal{I}$, be normalised so that null geodesics on $\mathcal{I}$ with initial endpoint at $t=0$ reconverge at $t=\pi$, and let $\{\mathcal{I} \mid t \in(T, T+\pi)\}=\mathcal{V}_{T}$. If $J^{+}\left(\mathcal{V}_{T}\right) \cap J^{-}\left(\mathcal{V}_{T}\right)$ is null geodesically complete, then the average mass on $\mathcal{V}_{T}$ is non-negative. If the matter flux near $\mathcal{V}_{T}$ vanishes as $\mathcal{O}\left(\Omega^{5}\right)$, the instantaneous mass is non-negative.

First, we must say what we mean by the mass, the average mass, and the matter flux. Ashtekar and Magnon ${ }^{(6)}$ define the following quantity associated with the timelike Killing field $\zeta^{a}=\frac{\partial}{\partial t}$ on $\mathcal{I}$.

$$
\mu=-\frac{1}{8 \pi} \int \Omega^{-1} C_{a b c d} n^{a} n^{c} \zeta^{b} d^{2} S^{d}=-\frac{1}{8 \pi} \int E_{a b} \zeta^{a} d^{2} S^{b},
$$

where the integral is over a two-sphere cross-section of $\mathcal{I}$. Here $d^{2} S^{a}=t^{a} d S^{2}$ where the measure $d S^{2}$ is that of the induced metric on the 2 -sphere (induced by the metric (1)), and $t^{a}$ lies in $\mathcal{I}$ and is a unit normal field for the 2-sphere (as computed using (1), of course). We may choose the cross-section orthogonal to $\frac{\partial}{\partial t}$, whence the integrand is $E_{00}$, the rescaled electric Weyl tensor.

The conservation law follows from the Bianchi identities, which Ashtekar and Magnon showed to imply that

$$
D^{a} E_{a b} \hat{=}-\frac{1}{2} \lim _{\rightarrow \mathcal{I}} \Omega^{-4} \mathcal{T}_{a}^{c} q_{c b} n^{a}
$$

where $q_{a b}$ is the restriction of the conformal metric $\tilde{g}_{a b}$ to $\mathcal{I}$ and $D_{a}$ is its compatible derivative. We may contract the free index with any conformal Killing field — in this case we use $\zeta^{a}=\frac{\partial}{\partial t}$ - and integrate over a region $V \subseteq \mathcal{I}$ bounded by a pair of 2 -sphere cross-sections $\mathcal{C}_{1}$ and $\mathcal{C}_{2}$ to obtain

$$
\left(\frac{-1}{8 \pi}\right) \int_{\mathcal{C}_{2}} E_{a b} \zeta^{a} d^{2} S^{b}-\left(\frac{-1}{8 \pi}\right) \int_{\mathcal{C}_{1}} E_{a b} \zeta^{a} d^{2} S^{b}=-\frac{1}{2} \int_{V} \lim _{\rightarrow \mathcal{I}} \Omega^{-4} n^{a} \mathcal{T}_{a}^{b} q_{b c} \zeta^{c}=F_{\zeta}(V)
$$

** This condition prohibits serious causality violations which may be due entirely to curvature deep in $\mathcal{M}$ and therefore cannot be controlled by restrictions placed on the asymptotic properties of the spacetime. 
The integral $F_{\zeta}(V)$ describes the matter flux (or flux of material energy) at ${ }^{\dagger} \mathcal{I}$ - there is no gravitational wave (Bondi) flux. ${ }^{(6)}$ Let us choose the cross-sections $\mathcal{C}_{1,2}$ to be the surfaces $t_{1}=$ const. and $t_{2}=$ const. We define the "mass at time $t$ " to be

$$
\mu(t)=\frac{-1}{8 \pi} \int_{\mathcal{C}(t)} E_{00} d^{2} S
$$

The prefactor $-\frac{1}{8 \pi}$ normalises $\mu$ to equal the mass parameter $m$ for the AdeS-Schwarzschild metric. Then the conservation law becomes

$$
\mu\left(t_{2}\right)-\mu\left(t_{1}\right)=F_{\zeta}(V)
$$

where $V$ is the region of $\mathcal{I}$ wherein $t_{1} \leq t \leq t_{2}$. Lastly, we define

$$
\langle\mu\rangle=\frac{1}{\pi} \int_{T}^{T+\pi} \mu(t) d t
$$

to be the average mass on the interval $t \in[T, T+\pi]$. This period corresponds to that used to quotient Universal anti-de Sitter space to obtain anti-de Sitter space, with its closed timelike curves.

We must also introduce the Borde energy condition, ${ }^{(17,7)}$ stated in Appendix 1. It is the weakest useful energy condition known to the author. Borde phrased his condition in terms of the Ricci tensor - the field equations are used to obtain the formulation quoted in the appendix. As the theorem stated there refers only to null geodesics, the cosmological term does not appear - the null Borde condition is not sensitive to the trace of the stressenergy tensor — which is a chief reason why much of this analysis passes over easily from ref. (8). Note the geodesic completeness condition is also weak; for example, it permits black holes and white holes.

The plan of the proof is as follows. We will look for a "fastest" causal curve joining two antipodal timelike generating curves of $\mathcal{I}$ (antipodal generators are those that are separated by arc length $\pi$ in the parameter $\sigma$ ). A putative fastest curve is any null curve that lies completely on $\mathcal{I}$ and joins these two timelike generators. However, if the mass is negative, we will be able to construct a faster curve which joins these two timelike generators by leaving $\mathcal{I}$ and passing through spacetime, whence it follows that the fastest such curve passes through spacetime as well, the existence of such a fastest curve being guaranteed by geodesic completeness. Being fastest, it cannot focus (i.e. it cannot have a

$\dagger$ The word "at" is incorrect, since the flux vanishes at $\mathcal{I}$, but it is perhaps descriptive. This integral actually describes flux through surfaces at arbitrarily large radii, and so perhaps "near" is a better word. 
conjugate pair), in contradiction to the Borde theorem.* The contradiction is avoided if the mass is non-negative on $\mathcal{I}$.

The expression " $t$-generator" will be used to refer to any one of the integral curves of $\frac{\partial}{\partial t}$ which also happens to lie on $\mathcal{I}$, since these curves generate $\mathcal{I}$. Now we are ready to prove the following proposition:

Proposition 3: Given that the assumptions of Theorem 2 hold for $\mathcal{V}_{T} \subseteq \mathcal{I}$, then

$$
\int_{0}^{\pi} E_{a b} \gamma^{a} \gamma^{b} d \sigma \leq 0
$$

along every null geodesic $\gamma$ ruling $\mathcal{V}_{T}$ and parametrised by $\sigma$ as defined above. ${ }^{\dagger}$

Proof: Let $P$ and $Q$ lie on antipodally opposed $t$-generators of $\mathcal{I}$, such that there is a null geodesic on $\mathcal{I}$ from $P$ to $Q$ (hence $P$ and $Q$ lie on the past and future boundaries, respectively, of $\mathcal{V}_{T}$ ). Because $P$ and $Q$ are on antipodal $t$-generators, the existence of one such geodesic implies the existence of a circle's worth (see fig.(4)), and there will be no faster curve on $\mathcal{I}$ from $P$ to the $t$-generator containing $Q$, so we select any one of these null geodesics and call it $\gamma$. Now consider that $\int_{0}^{\pi} E_{a b} \gamma^{a} \gamma^{b} d \sigma>0$ along $\gamma$. Then equation (42) for the time delay says that there is a null curve $\gamma^{\prime}$ (one of the ones permitted by our variational procedure) that joins $P$ to the $t$-generator of $Q$ by traversing not $\mathcal{I}$ but spacetime, which reaches that generator at some earlier point $Q^{\prime}$, in virtue of having a negative time delay.

In such a case, $Q$ will not lie on the achronal boundary set $\partial J^{+}(P)$, where $J^{+}(P)=J^{+}(P, \tilde{\mathcal{M}})$ denotes the set of points in $\left(\tilde{\mathcal{M}}, \tilde{g}_{a b}\right)$ reached by futurecausal curves from $P$. Say that this boundary meets $Q$ 's $t$-generator at $Q^{\prime \prime}$ in the chronological past of $Q$. Such a $Q^{\prime \prime}$ must exist, by the given condition that $\mathcal{I}$ contains a 2 -sphere cross-section which lies outside the future of $P$. It is a standard theorem (see Section 6.3 of ref. (10), for example) that one can trace back along the achronal boundary, obtaining a past-null geodesic, which has no endpoint except at $P$. This curve must enter the spacetime, for if it remained on $\mathcal{I}$ it could not do better than $\gamma$, which joins $P$ to $Q$, not $Q^{\prime \prime}$. Furthermore, this curve

* For a discussion of conjugate pairs, geodesic completeness, achronal boundaries, and related issues necessary for what follows, see ref. (10). Also, see fig. (5), which shows null geodesics leaving the future boundary of some point to enter into the interior, where they "focus", developing locii of conjugate points called caustics.

$\dagger$ Note this quantity also plays a key role in the discussions of Ashtekar and Penrose ${ }^{18}$ concerning ref. (7). 
must extend back to infinite negative values of its affine parameter, for otherwise it would have segments that are past-inextendible (having no past endpoint) null geodesics in the spacetime metric $g_{a b}$ and yet are of finite affine length. This cannot happen because $J^{+}\left(\mathcal{V}_{T}\right) \cap J^{-}\left(\mathcal{V}_{T}\right)$ is null geodesically complete. ${ }^{* *}$ Because it reaches $\mathcal{I}$ in the future, and extends to negatively infinite affine parameter values in the past, and never leaves the achronal boundary $\partial J^{+}(P)$, this geodesic is an infinite achronal geodesic of the spacetime metric $g_{a b}$.

However, the Borde theorem prohibits such a geodesic. Given the Borde energy condition, the Borde theorem guarantees that every infinite null geodesic has a conjugate pair separated by a finite affine length; i.e. every null geodesic experiences the lensing effect of the geometry and is "focussed". Any null geodesic from $P$ that has a conjugate pair cannot remain on the achronal boundary $\partial J^{+}(P)$ past the second of the two conjugate points of the pair, and therefore $\gamma^{\prime \prime}$ cannot have a conjugate pair. We have established a contradiction.

We note that the resolution of the contradiction, that $\int_{0}^{\pi} E_{a b} \gamma^{a} \gamma^{b} d \sigma \leq 0$ along each null geodesic $\gamma$ ruling $\mathcal{I}$ from $P$ to $Q$ (and for each $P$ and $Q$ there are a circle's-worth of such $\gamma$ ), implies that the $\gamma$ themselves are "fastest" curves (each being equally fast, of course). This is not in contradiction with the Borde theorem, of course, because these curves are not infinite achronal geodesics of the spacetime metric - they are of only finite affine length in the conformal metric on $\mathcal{I}$ and, in any case, they encounter neither matter nor generic curvature which could cause them to focus.

In order to prove Theorem 2 , it will be useful to reiterate some facts concerning $\mathcal{I}$. While $\mathcal{I}$ can be generated by integral curves of $\frac{\partial}{\partial t}$, it can also be generated by any family of a one-parameter set of families of null geodesics tangent to $\mathcal{I}$ (and indeed we have already made some use of these geodesics). Specifically, up to normalisation, a future-null vector field on $\mathcal{I}$ is given by specifying the parameter $\nu$ in

$$
\gamma^{a}=\frac{\partial}{\partial t}+\cos \nu \frac{\partial}{\partial \theta}+\frac{\sin \nu}{\sin \theta} \frac{\partial}{\partial \phi}
$$

Since $\mathcal{I}$ is a cylinder $R \times S^{2}$, we can fix a 2-sphere cross-section, say one of constant $t$, fix any $\nu$, and cover $\mathcal{I}$ by dragging the 2 -sphere along the resulting set of null integral curves. ${ }^{\dagger}$

** The reader might object that $P$ isn't in $\mathcal{V}_{T}$, but is merely on $\partial \mathcal{V}_{T}$, and therefore we cannot assume geodesic completeness. Having established $P$ and $Q^{\prime \prime}$, we could, however, move the whole argument up a little, pushing $P$ and with it $Q^{\prime \prime}$ slightly into the future, but making sure they both remain to the past of $Q$. The objection is then overcome.

$\dagger$ In some formulations, $\mathcal{I}$ or, in actual fact, $\mathcal{V}_{T}$, is a hyperboloid. Then these null 
Note that of course each family $\nu=$ const. consists therefore of a 2-sphere's-worth of null geodesics.

It is useful for future purposes to compute the average over $\nu$ at a point of $\mathcal{I}$ of $E_{a b} \gamma^{a} \gamma^{b}$

$$
\begin{aligned}
\int_{0}^{2 \pi} E_{a b} \gamma^{a} \gamma^{b} d \nu= & \int_{0}^{2 \pi}\left(E_{00}+2 E_{02} \cos \nu+2 E_{03} \frac{\sin \nu}{\sin \theta}+2 E_{23} \frac{\sin \nu \cos \nu}{\sin \theta}\right. \\
& \left.\quad+E_{22} \cos ^{2} \nu+E_{33} \frac{\sin ^{2} \nu}{\sin ^{2} \theta}\right) d \nu \\
= & \int_{0}^{2 \pi}\left(E_{00}+\frac{1}{2} E_{22}+\frac{1}{2 \sin ^{2} \theta} E_{33}\right) d \nu \\
= & \int_{0}^{2 \pi} \frac{3}{2} E_{00} d \nu \\
= & 3 \pi E_{00},
\end{aligned}
$$

where we have used the tracelessness of $E_{a b}$ in an intermediate step.

Proof of Theorem 2: Let $\mathcal{C}_{0}$ and $\mathcal{C}_{1}$ be two 2-sphere cross-sections of $\mathcal{I}$, say they are the surfaces $t=t_{0}$ and $t=t_{1}>t_{0}$ respectively, and let them bound a region $V \subseteq \mathcal{I}$. Then

$$
\begin{aligned}
\int_{V} d V \int_{0}^{2 \pi} E_{a b} \gamma^{a} \gamma^{b} d \nu & =3 \pi \int_{V} E_{00} d V=3 \pi \int_{t_{0}}^{t_{1}} d t \int_{\mathcal{C}(t)} E_{00} \sin \theta d \theta d \phi \\
& =-24 \pi^{2} \int_{t_{0}}^{t_{1}} \mu(t) d t,
\end{aligned}
$$

where $\mathcal{C}(t)$ is the 2 -sphere cross-section of $\mathcal{I}$ defined by $t_{0} \leq t=$ const. $\leq t_{1}$ and where $\mu(t)$ is the mass on that 2 -sphere, as given by evaluating equation (43) or, equivalently, (46).

geodesic generators have an interesting historical connection. They form a circle's-worth of families of null geodesic generators of the 3-dimensional hyperboloid, and this is a direct generalisation of the observation, attributed ${ }^{(19)}$ to the geometer and architect Sir Christopher Wren, that a 2-dimensional hyperboloid is generated by either of two families (i.e. an $S^{0}$ 's-worth of families) of straight lines. 
Now rewrite the integral above making use of the null generators. Specifically, let $t_{0}=T$ and choose $t_{1}$ such that $\mathcal{C}_{1}$ can be reached from $\mathcal{C}_{0}$ by traversing a null generator from $\sigma=0$ to $\sigma=\pi$ (this distance is independent of the value of $\nu$ for the chosen null generator, since the surfaces $\mathcal{C}_{0}$ and $\mathcal{C}_{1}$ are surfaces of constant $t$ ). Then $V=\mathcal{V}_{T}$ and

$$
\begin{aligned}
\int_{\mathcal{V}_{T}} d V \int_{0}^{2 \pi} E_{a b} \gamma^{a} \gamma^{b} d \nu & =\int_{0}^{2 \pi} d \nu \int_{\mathcal{V}_{T}} E_{a b} \gamma^{a} \gamma^{b} d V \\
& =\int_{0}^{2 \pi} d \nu \int_{\mathcal{C}^{\prime}} \sin \theta d \theta d \phi \int_{0}^{\pi} E_{a b} \gamma^{a} \gamma^{b} d \sigma
\end{aligned}
$$

where $\mathcal{C}^{\prime}$ is also a 2 -sphere, but this time it is the 2-sphere of null generators for some fixed $\nu$. Now we have established in Proposition 3 that the inner integral in equation (52) is bounded above by 0 on the region of integration $\sigma \in[0, \pi]$, provided of course the stated assumptions hold on $\mathcal{V}_{T}$. Writing this result as

$$
\int_{0}^{\pi} E_{a b} \gamma^{a} \gamma^{b} d \sigma \leq-b_{\gamma} \leq 0
$$

where the bound $b_{\gamma}$ can depend on $\gamma$, then equation (52) gives

$$
\int_{\mathcal{V}_{T}} d V \int_{0}^{2 \pi} E_{a b} \gamma^{a} \gamma^{b} d \nu \leq \int_{0}^{2 \pi} d \nu \int_{\mathcal{C}^{\prime}}\left(-b_{\gamma}\right) \sin \theta d \theta d \phi \leq 0 .
$$

By combining equations (46), (48), (50), and (54), we obtain

$$
\langle\mu\rangle=\frac{1}{\pi} \int_{T}^{T+\pi} \mu(t) d t \geq 0 .
$$

This is the required result for the average mass. The result for the instantaneous mass derives from the conservation law (47) when the matter flux at $\mathcal{V}_{T}$ vanishes.

\section{Conclusions}

The theorem is not a true positivity theorem in the usual sense. Setting aside for the moment the question of the time-averaged mass, we still have only a non-negativity theorem. In contrast to the hypersurface spinorial argument ${ }^{(4)}$, it does not imply that exact 
AdeS spacetime is the unique ground state, leaving open the possibility of an instability of AdeS spacetime via quantum tunnelling between AdeS spacetime and another zero-energy state, perhaps one that obeys the Borde energy condition, but not the dominant energy condition, so as not to violate the theorem of ref. (4).

It is no surprise that the theorem refers to time-averaged mass when there is matter flux at $\mathcal{I}$. After all, the Borde energy condition also refers to time-averages of the matter tensor. It is quite reasonable that the consequence of having no null geodesic encounter net negative energy when averaged over its length (a weak paraphrasing of the Borde condition) is that the mass, averaged over time, should be positive. The time average is unnecessary in the case of asymptotically flat spacetimes because their null boundaries prevent what is entirely possible in the asymptotically AdeS case, that there can be inbound fluxes of matter energy near $\mathcal{I}$ which, by being present in sufficient quantity, change the sign of the total mass from what it was previously.

Although the asymptotic structure used here was that of Universal AdeS spacetime (see part (ii) of Definition 1), the argument clearly goes through in any quotient spacetime whose universal cover is asymptotically AdeS in the sense of the Definition. If the covering spacetime admits an infinite achronal geodesic, as it will if the mass is negative, then this geodesic has no conjugate pair. It will project under the covering map to a null geodesic in the quotient spacetime, and this geodesic will also lack a conjugate pair (although it will no longer be achronal). It will therefore remain in contradiction with the Borde theorem.

It is not possible to take the $\Lambda \rightarrow 0$ limit of the argument herein to obtain a positivity proof for the mass of an asymptotically flat spacetime. Such a limit may be described as singular, and several steps do not go through. The topology of $\mathcal{I}$ is changed significantly, due to the singularity at the point $i^{0}$ representing spatial infinity. As well, such a limit would fail to admit gravitational radiation at $\mathcal{I}^{+}$.

However, it is very likely that methods quite similar to those discussed here will work in the other case, that of positive cosmological constant. This is often referred to as the asymptotically de Sitter case, but because the boundary-at-infinity for a de Sitter universe is comprised solely of two spacelike surfaces (called timelike infinity), the term usually refers instead to universes which asymptote to a positive spatial curvature Robertson-Walker metric that describes the region of de Sitter space inside of a cosmological horizon. ${ }^{(9,10)}$ Ref. (9) discusses the McVittie metric, which describes an isolated mass embedded in a Robertson-Walker background spacetime. It is very straightforward to apply the method of ref. (11) to the McVittie case and relate positivity of the time delay to positivity of the McVittie mass parameter. I will return to this case in future work.

Lastly, note the similarlity of the integral condition in Proposition 3 governing the time delay (equivalently, the left-hand-side of equation (53)) to the Averaged Null Energy Condition, i.e. to the Borde Condition (A.1.1) in the case where the null geodesic path of 
integration is extended all the way to $\mathcal{I}$. $^{*}$ It is conceivable that this may be suggestive of a quasi-local energy construction based on the electric components of Weyl defined with respect to and integrated over appropriate timelike 3-surfaces (which could be projected down to spacelike 2-surfaces when the flux across the surface vanished), and that such a construction might have certain desirable properties, such as positivity, at least when appropriate. We note that the idea that Weyl curvature describes in a quasi-local way the contribution of the gravitational field to the energy is a long-established one. ${ }^{(20)}$

\section{Acknowledgements}

I thank Malcolm MacCallum for communications concerning the use of the computer program RCLASSI, which was used to check equations (22) and to obtain equations (28). I thank the relativity and cosmology group of Dalhousie University for discussions and technical assistance with RCLASSI and with production of the figures, Abhay Ashtekar and Jorge Pullin for relevant correspondence, and my co-authors in ref. (7) for permission to reproduce one of the figures from it and for discussions on topics related to those appearing herein.

\section{Appendix 1: Borde's Theorem}

Borde's paper ${ }^{(17)}$ actually discusses focussing theorems for both timelike and null curves. Herein, we state a theorem for null curves alone, and we use the field equations to express the integral condition in terms of the matter tensor instead of the Ricci curvature.

Borde's Focussing Theorem: Let $\gamma(t)$ be a complete affinely parametrised causal geodesic with tangent $\ell^{a}$ and let $\ell_{[a} R_{b|c d| e} \ell_{f]} \ell^{c} \ell^{d} \neq 0$ somewhere on $\gamma$ (this is known as the null generic condition). Suppose that for any $\epsilon>0$ there is a $b>0$ such that for any $t_{1}<t_{2}$ there is a pair of intervals $I_{1}<t_{1}$ and $I_{2}>t_{2}$ of lengths $\geq b$ such that

$$
\int_{t^{\prime}}^{t^{\prime \prime}} T_{a b} \ell^{a} \ell^{b} d t \geq-\epsilon \quad \forall t^{\prime} \in I_{1}, \forall t^{\prime \prime} \in I_{2} .
$$

Then $\gamma$ contains a pair of conjugate points.

In the text, the integral (A.1.1) is referred to as the Borde energy condition.

\section{Appendix 2: The AdeS-Schwarzschild Solution}

\footnotetext{
* I thank Gholamhossein Abolghasem for bringing this similarity to my attention.
} 
The AdeS-Schwarzschild solution is an exact solution whose metric can be written as

$$
\begin{aligned}
d s^{2} & =-\left(1-\frac{1}{3} \Lambda r^{2}-\frac{2 m}{r}\right) d t^{2}+\frac{d r^{2}}{\left(1-\frac{1}{3} \Lambda r^{2}-\frac{2 m}{r}\right)}+r^{2}\left(d \theta^{2}+\sin ^{2} \theta d \phi^{2}\right), \\
& =-\left(1+r^{2}-\frac{2 m}{r}\right) d t^{2}+\frac{d r^{2}}{\left(1+r^{2}-\frac{2 m}{r}\right)}+r^{2}\left(d \theta^{2}+\sin ^{2} \theta d \phi^{2}\right),
\end{aligned}
$$

where we have rescaled $s, r, t$, and $m$ by $\sqrt{-3 / \Lambda}$, recalling that of course $\Lambda<0$. We may now choose

$$
\Omega^{2}=\frac{1}{1+r^{2}}
$$

The metric becomes

$$
\begin{aligned}
d s^{2}= & \Omega^{-2}\left\{-d t^{2}+\frac{d \Omega^{2}}{1-\Omega^{2}}+\left(1-\Omega^{2}\right)\left(d \theta^{2}+\sin ^{2} \theta d \phi^{2}\right)\right. \\
& \left.+2 m \Omega^{3}\left(d t^{2}+d \Omega^{2}\right)+\mathcal{O}\left(\Omega^{4}\right)\right\} \\
= & \Omega^{-2} d \tilde{s}^{2},
\end{aligned}
$$

where $d \tilde{s}^{2}$ is the conformal metric. We read off $B=0$ and $K=2 m$, so we perform the coordinate transformation

$$
\Omega=\bar{r}-\frac{1}{4} m \bar{r}^{4}
$$

Then the conformal metric becomes

$$
d \tilde{s}^{2}=-d t^{2}+\frac{d \bar{r}^{2}}{1-\bar{r}^{2}}+\left(1-\bar{r}^{2}\right)\left(d \theta^{2}+\sin ^{2} \theta d \phi^{2}\right)+2 m \bar{r}^{3} d t^{2}
$$

This can be written in the form

$$
\begin{aligned}
d \tilde{s}^{2}=(1- & \left.\frac{2}{3} m \bar{r}^{3}\right)\left[-d t^{2}+\frac{d \bar{r}^{2}}{1-\bar{r}^{2}}+\left(1-\bar{r}^{2}\right)\left(d \theta^{2}+\sin ^{2} \theta d \phi^{2}\right)\right] \\
& +\frac{2}{3} m \bar{r}^{3} d \bar{r}^{2}+\frac{2}{3} m \bar{r}^{3}\left(2 d t^{2}+d \theta^{2}+\sin ^{2} \theta d \phi^{2}\right)+\mathcal{O}\left(\bar{r}^{4}\right)
\end{aligned}
$$

suggestive of equation (31). A simple computation (with RCLASSI) confirms that $E_{00}=$ $-2 m, E_{22}=-m$, and of course $E_{33}=-m \sin ^{2} \theta$ for this metric. Thus, we recover the form (31). We may now transform to $\bar{\xi}=\operatorname{Arccos} \bar{r}$ and write

$$
d \tilde{s}^{2}=\left(1-\frac{2 m}{3} \cos ^{3} \bar{\xi}\right) d \tilde{s}_{\mathrm{AdeS}}^{2}-\frac{2}{3}\left(\cos ^{3} \bar{\xi}\right) E_{i j} d x^{i} d x^{j}+\frac{2 m}{3} \cos ^{3} \bar{\xi} d \bar{\xi}^{2}+\mathcal{O}\left(\cos ^{4} \bar{\xi}\right)
$$

If $m>0$, then these metrics have event horizons which do not intersect $\mathcal{I}$. If $m<0$, no event horizons exist, and observers at $\mathcal{I}$ can always see into the singularity, so a region $\mathcal{V}_{T}$ obeying the geodesic completeness and other criteria required herein cannot be found. 


\section{References}

(1) Breitenlohner, P., and Freedman, D.Z., Ann. Phys. 144, 249 (1982).

(2) Abbott, L.F., and Deser, S., Nuc. Phys. B 195,76 (1982).

(3) Witten, E., Commun. Math. Phys. 우, 381 (1981).

(4) Gibbons, G.W., Hull, C.M., and Warner, N.P., Nuc. Phys. B $\underline{218}, 173$ (1983).

(5) Hawking, S.W., Phys. Lett. 126B, 175 (1983).

(6) Ashtekar, A., and Magnon, A., Class. Quantum Gravit. 1, L39 (1984).

(7) Penrose, R., Sorkin, R.D., and Woolgar, E., preprint gr-qc/9301015 (1993).

(8) Gibbons, G.W., and Wells, C.G., DAMTP preprint R93/25 (1993), available as grqc/9310002.

(9) Kastor, D., and Traschen, J., University of Massachusetts preprint UMHEP-399 (1993), available as gr-qc/9311025.

(10) Hawking, S.W., and Ellis, G.F.R., The Large Scale Structure of Spacetime (Cambridge University Press, Cambridge, 1973).

(11) Woolgar, E., preprint to appear in Proc. Fifth Can. Conf. on Gen. Rel. and Rel. Astrophys., held at Waterloo, May 1993.

(12) Chruściel, P.T., MacCallum, M.A.H., and Singleton, D.B., preprint gr-qc/9305021 (1993), and references therein.

(13) Penrose, R., Proc. Roy. Soc. (London) A로, 159 (1965).

(14) Wald, R.M., General Relativity (University of Chicago Press, Chicago, 1984).

(15) Beig, R., and Schmidt, B.G., Commun. Math. Phys. 87, 65 (1982).

(16) See Nityananda, R., and Samuel, J., Phys. Rev D $\underline{45}, 3862$ (1992) for a discussion of this variation and its application to Fermat's Principle in General Relativity.

(17) Borde, A., Class. Quantum Gravit. 4, 343 (1987); see also Tipler, F.J., J. Diff. Eq. 30, 165 (1978); Phys. Rev. D $\underline{17}, 2521$ (1978).

(18) Ashtekar, A., and Penrose, R., Twistor Newsletter $\underline{31}, 1$ (1990).

(19) Rouse Ball, W.W., A Short Account of the History of Mathematics, $4^{\text {th }}$ ed. (1908) (reprinted by Dover Press, New York, 1960).

(20) Penrose, R., Perspectives in Geometry and Relativity (Hlavaty Festschrift), ed. B. Hoffmann, P. 259 (Indiana University Press, Bloomington, 1966). 


\section{Figure Captions}

Fig. 1: Anti-de Sitter spacetime embedded in the Einstein cylinder. The ruled region is the image of the AdeS spacetime, while the vertical lines constitute the boundary $\mathcal{I}$. Universal AdeS spacetime is represented by an infinite vertical strip on this diagram.

Fig. 2: $\gamma$ and $\gamma^{\prime}$. Here $\gamma$ is ruling $\mathcal{I}$, while $\gamma^{\prime}$ moves alittle into spacetime. The "neighbourhood of infinity" in which $\gamma^{\prime}$ is constrained to move is represented by the region between the outer cylinder $(\mathcal{I}$ itself $)$ and the inner cylinder. In the case drawn here, the time delay of $\gamma^{\prime}$ is positive.

Fig. 3: This traces the progress of $\gamma$ and $\gamma^{\prime}$ through space (actually, space plus boundary) at successive intervals of time. Space here is a hemisphere, which would be accurate for the exact AdeS case. The boundary (infinity) is the equator $\xi=\frac{\pi}{2}$, and $\gamma$ moves along this equator while $\gamma^{\prime}$ moves through spacetime near the equator. The coordinates $\xi$ and $\phi$ are shown; $\theta$ is suppressed.

Fig. 4: This shows successive $t=$ const. slices of $\mathcal{I}$. Since $\mathcal{I}=S^{2} \times R$, no coordinate is suppressed. From any point $p \in \mathcal{I}$, there originates a circle's-worth of null geodesics which rule $\mathcal{I}$. Each of these geodesics traverses the $S^{2}$ and arrives at the antipodal point at the same time as all the others. This sequence of sketches tracks their progress.

Fig. 5: A null cone of a point $p$ in a generic curved spacetime on which the Borde energy condition holds. The null geodesics are deflected by the curvature and begin to converge upon conjugate points which lie along the caustics, which are the cuspoidal structures in the light cone's interior. They first pass through a "crossing region" and leave the boundary of the future of $p$. 